\title{
Lebensraum Hecken
}

\section{Stephanie Seitz}

\begin{abstract}
Hedges are important for people and many animals. The development and construction will be described. Some typical hedge plants such as blackthorn and hawthorn are briefly introduced.
\end{abstract}

\section{Zusammenfassung}

Hecken sind wichtig für Menschen und viele Tieren. Ihre Entwicklung und Aufbau werden beschrieben. Einige typische Heckengehölze wie Schlehe und Weißdorn werden kurz vorgestellt.

\section{Bedeutung von Hecken früher}

Erste archäologische Hinweise lassen darauf schließen, dass schon vor 6000 Jahren Menschen ihre Ackerflächen mit Hecken umpflanzten. Hecken hatten schon zur damaligen Zeit die Aufgabe der Abgrenzung von Feldern und Viehweiden und kennzeichneten Grundstücksgrenzen (Kurz, MachatscheK \& Igelhauser 2011).
Häufig dienten sie auch als Windschutz. Sie waren wichtige Lieferanten von Laubfutter und Holz, hatten aber auch Verteidigungsfunktion, denn dichte dornig-stachelige Hecken sind oft undurchdringbar. Der Stamm der Nervier beispielsweise pflanzte dichte Dornenhecken gegen Reiterangriffe. Nervier waren ein in der Antike lebender belgischer Volksstamm.

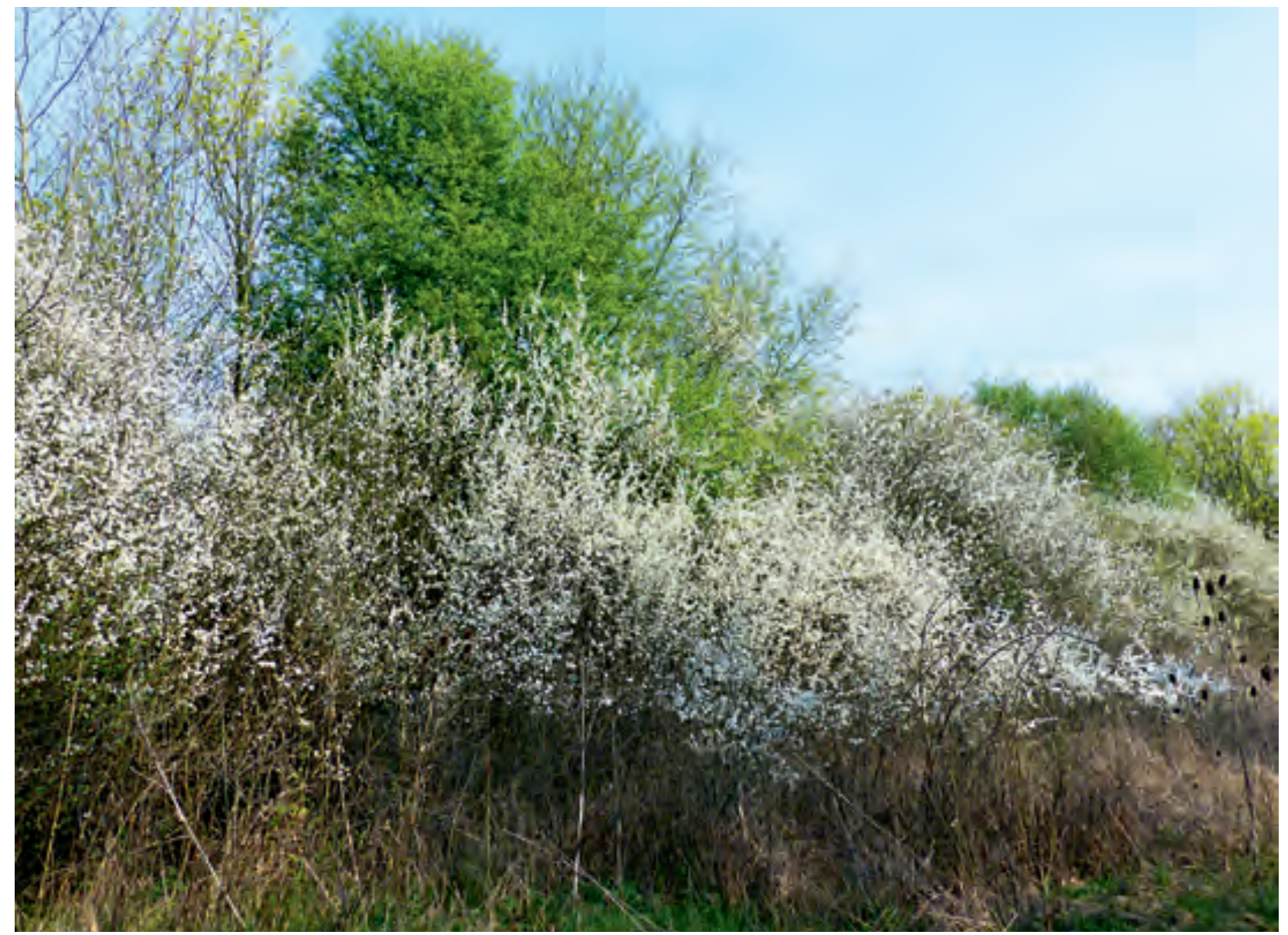

Abb. 1: Blühende Schlehenhecke im Rhein-Main-Gebiet an der Nidda. (Foto: H. Steinecke) 


\section{Um Heckenpflanzen ranken sich viele Mythen und Legenden:}

„Die Hagrose, Heckenrose, Hagedorn (Rosa canina) hat ihren Namen daher, weil sie mir ihren dornigen (Anmerkung: was hier als Dornen bezeichnet wird, sind botanisch korrekt Stacheln) Zweigen einen so dichten Haag bildet, daß weder Mensch noch Thier durch kann. Sie wächst besonders gern da, wo früher heilige Haine standen, oder an Plätzen, die einst zu Opfer- und Begräbnisstätten gedient hatten. Sie heißt noch in manchen Gegenden, z. B. am Niederrhein: Friggborn (Friggaborn) und darf nur am Freitag (Tag der Freja oder Frigga) gepflückt werden. Die Bedeutung der Blumen sagt von der Hagrose: Wer etwas Liebes hat, daß ihm in allen Dingen gefällt, von dem er aber nichts erzählen darf, der soll Hagedorn tragen, denn der hat die Art, daß er, wie freundlich er auch aussehen mag, sich wegen seiner Dornen noch nicht anrühren läßt ...

Es war im Jahre 1090, als der Graf von Berge seine Frau, die er für untreu hielt, umbrachte und ihre Kinder in der Wildnis aussetzte, damit sie von Wölfen und Bären zerrissen würden. Allein die hl. Maria hatte Erbarmen mit den Kleinen und zog einen so dichten Haag von wilden Rosen um sie, daß kein Raubthier ihnen nahen konnte. Als der Graf auf einer Jagd seine Kinder unversehrt wiedersah, erkannte er sein Unrecht, nahm die Kleinen wieder zu sich und wandelte zum Zeichen seiner Reue, das goldene Scepter seines Wappens in eine Hagerose" (Perger 1864, S. 235, 237).

Die Flurbezeichnung Hag oder Haag ist ein von einer Hecke umgebenes Gelände. Noch heute weisen Städtenamen wie Hagen, Steinhagen oder Den Haag auf diese Siedlungsform hin. Bei Kelten und Germanen galten siedlungsnahe Hecken als Schutzwall, der die Menschen auch vor bösen Geistern beschützen sollte. Durchlässe in Hecken

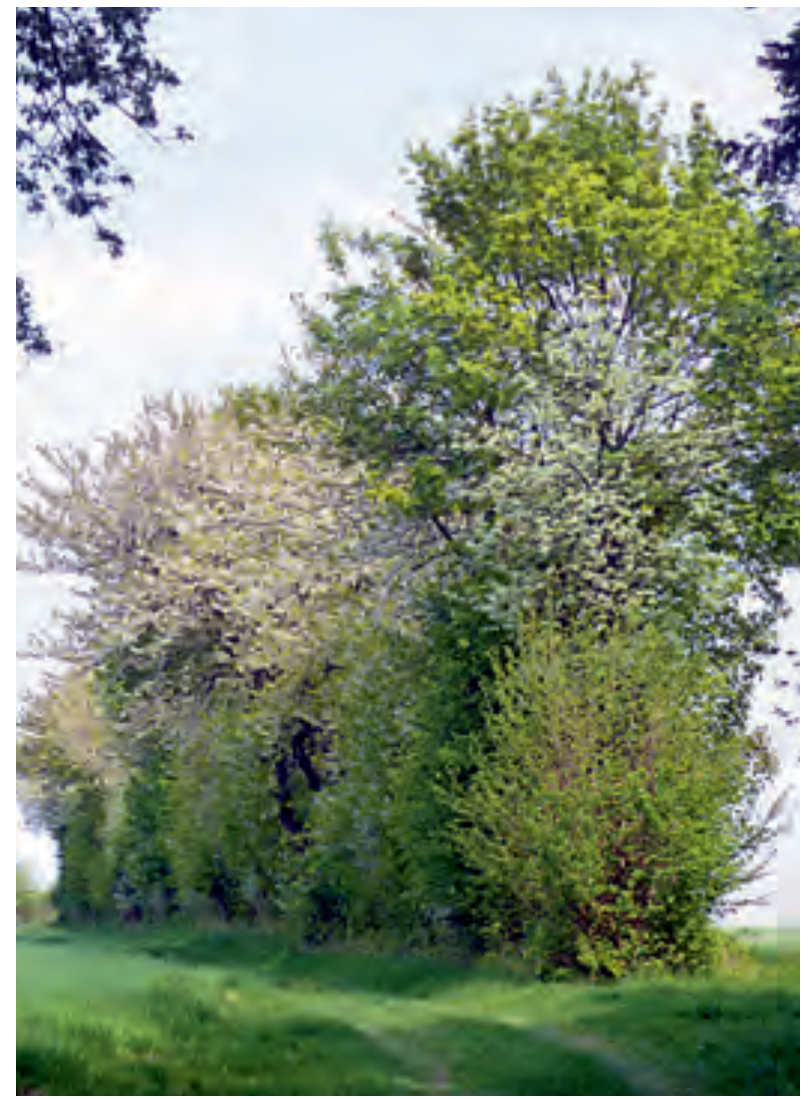

Abb. 2: Eine verschiedene Baumarten (Vogel-Kirsche, Mehlbeere, Berg-Ahorn) enthaltene Baumhecke am Mittelrhein. (Foto: H. Steinecke)

wurden als Tore in die Feen- oder Unterwelt interpretiert. Auf einen Hag beziehen sich die englische Bezeichnung hag (Hexe) sowie die alten Namen Hagetisse und Hagazussa (= Zaunreiterin) für eine Hexe bzw. eine Frau, die sich im Gebüsch aufhält (SChubert 2004). Typische Heckenpflanzen tragen deshalb den Wortstamm „hag“ in ihrem Namen wie beispielsweise Hagedorn (Weißdorn, Crataegus), Hagerose bzw. ebenfalls Hagedorn (Hunds-Rose, Rosa canina), Hagebutte (die Früchte der Rose), oder Hagebuche (Hainbuche, Carpinus betulus).

Der Schutz durch Hecken wurde bis ins 17. Jahrhundert beibehalten. Gräben, die mit Dornenhecken bepflanzt wurden, nannte man Landwehr. Sie sollten die naheliegenden Siedlungen vor Angriffen durch Feinde schützen und Territorien abgrenzen. Um einen besonders dichten Wuchs zu erzeugen, wurden junge Triebe nach unten gebogen (,gebückt“) und waagerecht in der Erde befestigt, wo sie dann Wurzeln bilden und 


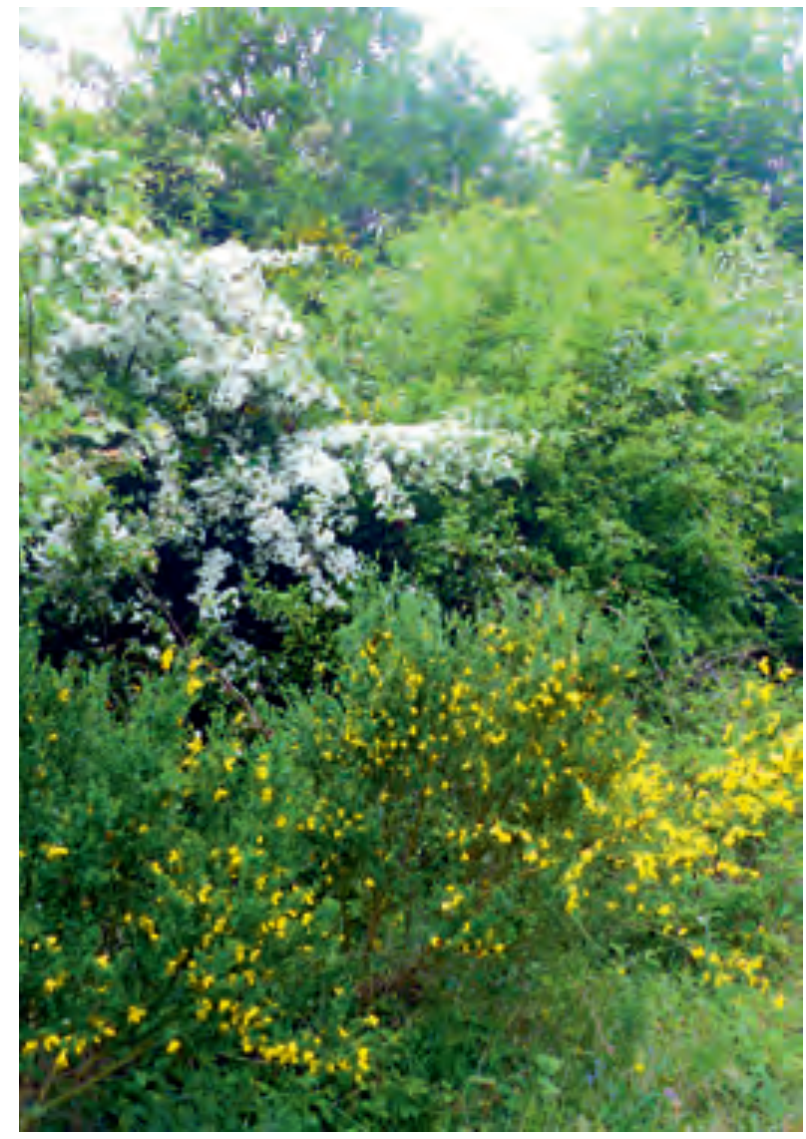

Abb. 3: Frühsommerlicher Aspekt einer Hecke mit Besenginster und Weißdorn an der Nahe. (Foto: H. Steinecke)

weiter wachsen konnten. Häufig wurden dabei junge Hainbuchen miteinander verflochten, so dass sie mit der Zeit ein dichtes, so genanntes Gebück bildeten. Wer im Taunus wandert, stößt möglicherweise auf den ausgeschilderten Rheingauer Gebückweg. Das aus „gebückten“ Bäumen bestehende Rheingauer Gebück umgab mehrere Jahrhunderte lang bis zum Ende des 18. Jahrhunderts den Rheingau.

Hecken sind seit dem letzten Jahrhundert bei uns seltener geworden. Ab den 1950er Jahren wurden viele von ihnen gerodet, um landwirtschaftliche Flächen effektiver bearbeiten zu können (LfL 2015). Sie werden heute in der freien Landschaft nicht mehr so häufig neu angelegt. Statt Dornengehölze sind heutzutage Stacheldrahtund Elektrozäune weit verbreitet, um vor allem Weideland einzugrenzen. Beliebter sind Hecken dagegen im Privatbereich zur Umgrenzung von Gartengrundstücken. Sie dienen dann vor allem dem Sicht- und Lärmschutz. Sie tragen aber auch zu einem günstigeren, ausgeglichenerem Mikroklima bei.

\section{Hecke, Gebüsch und Feldgehölz}

Sträucher und Bäume erscheinen landschaftsprägend in verschiedenen Größen und Gruppierungen. Dabei werden kleine Gruppen aus jungen Bäumen und Sträuchern mit einer flächigen Ausdehnung als Gebüsch bezeichnet. Feldgehölze sind Gebüsche, bei denen die Baumgruppen zu kleinen Wäldern heranwachsen. In der Forstwirtschaft gelten sie aber nicht als richtige Wälder, da Feldgehölze weniger als einen Hektar Fläche einnehmen. Hecken sind linienförmig wachsende Sträucher und einzelne Bäumen mit unterschiedlicher Nutzung sowie Artenzusammensetzung.

\section{Aufbau und Entwicklung einer Hecke}

Hecken lassen sich in vier unterschiedliche Bereiche untergliedern. Um den Heckenkern hüllt sich an den Seiten die Wandzone und darüber die Decke. Am Fuße bildet sich der Heckensaum, der auch Krautsaum genannt wird.

Heckensäume setzen sich je nach Standort aus mehrjährigen Gräsern und Kräutern unterschiedlicher Gattungen und Arten zusammen. Sie bieten vielen Pflanzen und Tieren, darunter zahlreichen Insekten, einen Lebensraum. Häufig ist dort auch die Brennessel anzutreffen, die nicht nur für verschiedene Schmetterlingsraupen (z. B. Kleiner Fuchs, Pfauenauge) eine essentielle Futterpflanze darstellt. Durch zweimaliges Mähen im Zeitraum von drei bis fünf Jahren werden Heckensäume gepflegt. Dabei werden aufkommende, mit den Hecken konkurrierende Gehölze unterdrückt.

Wesentliche Faktoren für die Entwicklung und Zusammensetzung von Hecken sind Lichteinfall, Temperatur, Bodenfeuchte, Verdunstungsrate und das regelmäßige Auftreten starker Winde. Den äußeren Schichten der Hecke steht naturgemäß das meiste Licht zu Verfügung, je nach Dichte der Hecke erreicht die Lichtintensität im Innern der Hecke nur noch wenige Prozent der ursprünglich auf die Hecke treffenden Lichtintensität. Da zu- 


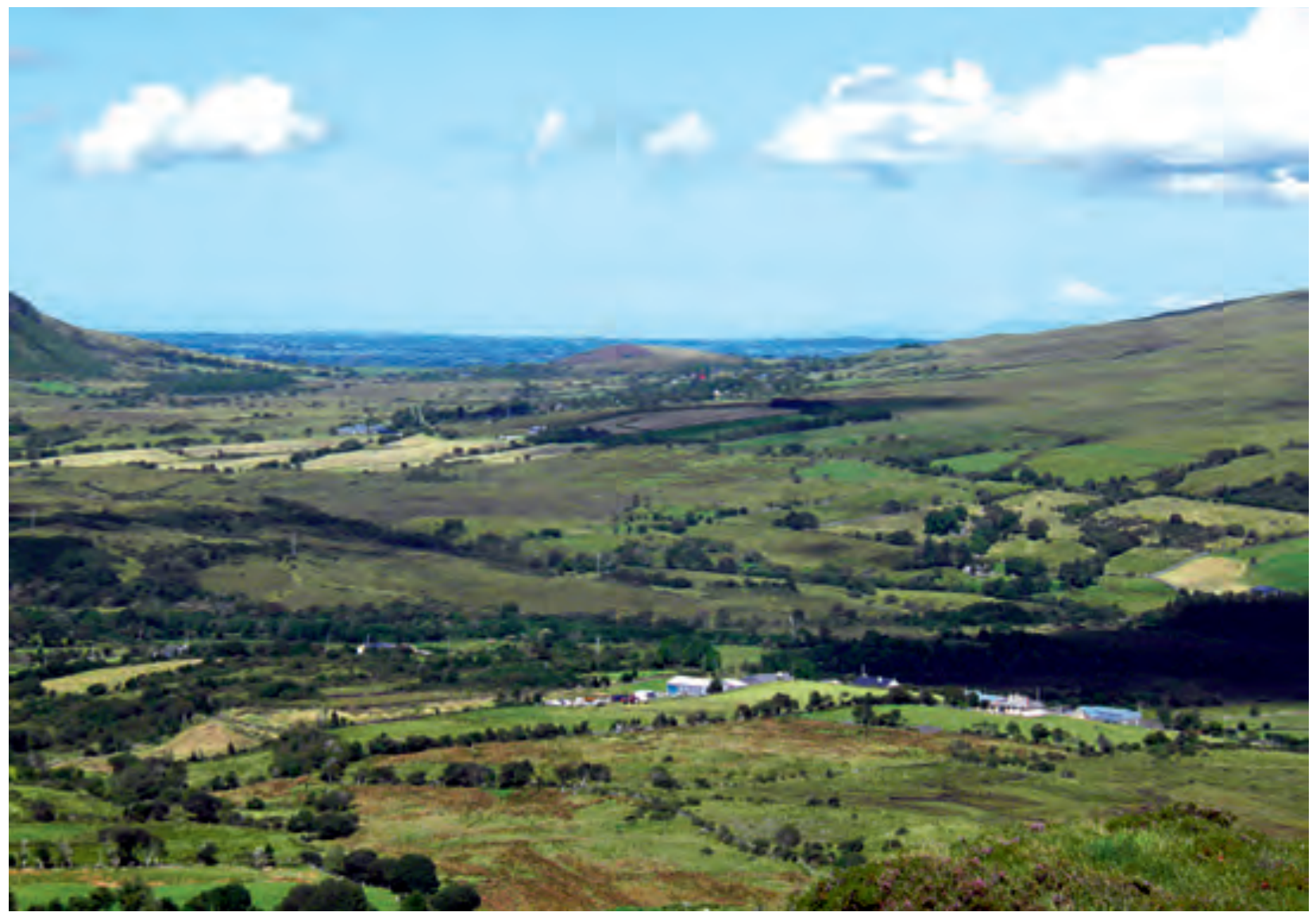

Abb. 4: Typische Heckenlandschaft in Irland. (Foto: K. Steinecke)

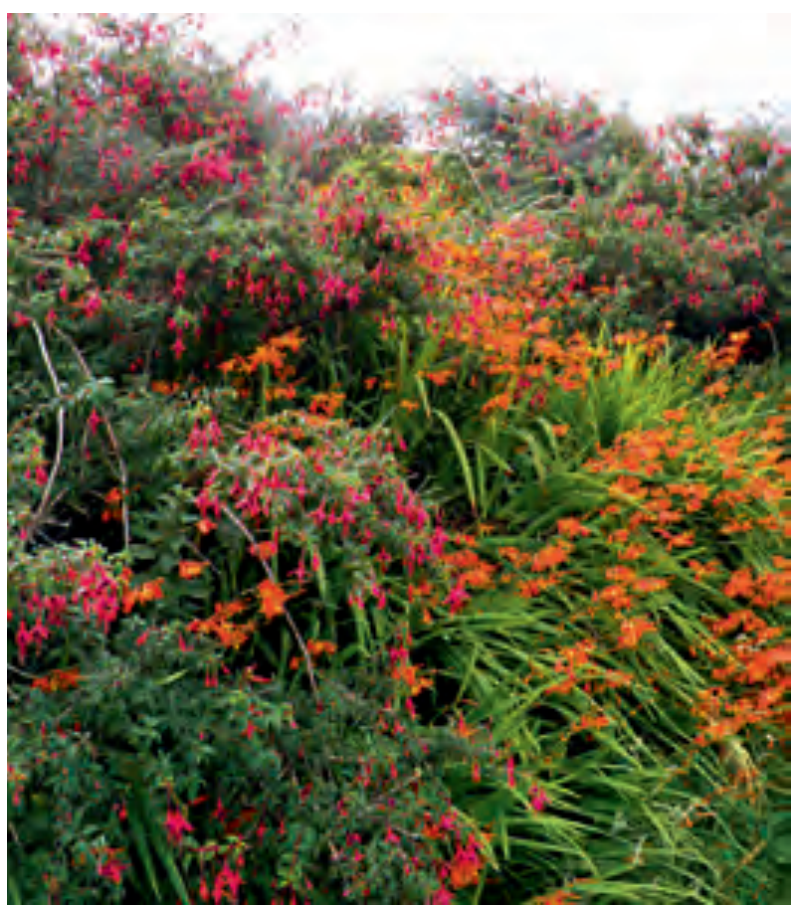

Abb. 5: Abhängig von den klimatischen Gegebenheiten siedeln sich unterschiedliche Arten in einer Hecke an. Hier ein Ausschnitt einer für Irland typischen Hecke mit verwilderten Fuchsien und Montbretien, beides Neophyten in Irland.

(Foto: K. STEINECKe) dem die Südseite einer Hecke insgesamt in einen höheren Lichtgenuss kommt als ihre Nordseite, sind ganz allgemein die Lichtverhältnisse innerhalb einer Hecke sehr variabel. Kräuter und junge Sträucher können sich im Inneren einer Hecke kaum ansiedeln, da es dort zu dunkel ist.

Die Entwicklung einer Hecke läuft in vier Phasen ab, nämlich der Pionier-, Anreicherungs-, Reife- und Abbauphase. In der Pionierphase siedeln sich zunächst bevorzugt solche Arten an, die nicht verbissen werden wie beispielsweise die stechenden Gewächse Brombeere, Schlehe, Weißdorn und Hundsrose. Diese Arten werden meist durch Vögel ausgebreitet, die die Früchte fressen und dabei die Samen andernorts wieder ausscheiden. Durch Bildung von Ausläufern kann sich die Hecke nach und nach verbreitern. Je größer die Hecke ist, um so mehr Tiere, darunter auch diverse Vögel, halten sich dort auf und suchen dort Schutz oder Brutplätze. Über den Kot der Vögel kommen in der Anreicherungsphase immer mehr Pflanzenarten dazu. Im Schutze der bereits vor- 


\section{Typische Heckengehölze}

Gemeiner Liguster (Ligustrum vulgare); bis 15 m hoch; weiße Blüten im Juni und Juli; schwarze Beerenfrüchte; für den Randbereich einer Hecke geeignet; giftig.

Holunder (Sambucus nigra); bis $7 \mathrm{~m}$ hoch; weiße doldenförmige Blütestände erscheinen im Mai; blauschwarze Früchte reifen ab August; Früchte roh giftig, aber zu Saft oder Gelee geeignet.

Haselnuss (Corylus avellana); bis $6 \mathrm{~m}$ hoch; Kätzchenblüher; blüht je nach Standort schon ab Januar vor dem Laubaustrieb; Nussfrüchte reifen ab September; gut zurückschneidbar und treibt schnell wieder aus.

Schlehe (Prunus spinosa); bis $3 \mathrm{~m}$ hoch; weiße Blüten erscheinen ab März; gute Bienenweide, Früchte dunkelblau und pflaumenartig; reifen ab Oktober, sind aber erst nach Frost genießbar, da ansonsten zu sauer und herb.
Eingriffeliger Weißdorn, Hagedorn (Crataegus mongyna); meist bis $6 \mathrm{~m}$ hoch; Blütezeit im Mai und Juni; rote Apfelfrüchte; essbar, aber herb; Sprossdornen, diese ideal für den Neuntöter, um seine Beute aufzuspießen; Blätter sind Futter z. B. für den Segelfalter.

Gewöhnlicher Schneeball (Viburnum opulus); bis $6 \mathrm{~m}$ hoch, weiße doldenartige Blütenstände ab Mai; rote beerenartige Steinfrüchte reifen ab August bis November, Wintersteher, wichtige Futterquelle für Vögel im Winter; abgeschnittene Zweige wurzeln schnell an.

Eibe (Taxus baccata); bis $15 \mathrm{~m}$ hoch; Blütezeit von März bis Mai; roter Samenmantel essbar, ansonsten stark giftig und deshalb nicht für Grundstücke mit kleinen Kindern geeignet; Eiben können bis 1000 Jahre alt werden; Rückschnitt bis ins alte Holz möglich. handenen Dornensträucher können sich in der folgenden Reifephase nun auch verbissempfindliche Gehölze ansiedeln. Hierzu zählen beispielsweise Pfaffenhütchen und Holunder. Die schützenden Dornensträucher in einer artenreichen Hecke werden Ammengehölze genannt. In der Abbauphase werden lichthungrige Gehölze immer mehr an den Rand der Hecke verdrängt, bis diese schließlich nur noch im Heckenmantel zu finden sind oder später sogar komplett ausfallen. Die Gewöhnliche Berberitze (Berberis vulgaris) ist ein Strauch, der nur an sonnigen bis halbschattigen Standorten gedeiht, reine Schattenstandorte meidet und deshalb bevorzugt in Gebüschen, an Hecken- oder Waldrändern anzutreffen ist.

Je nach Höhe der Hecke werden bis $3 \mathrm{~m}$ hohe Niederhecken, bis $5 \mathrm{~m}$ hohe Hochhecken und mit Bäumen durchsetzte Baumhecken unterschieden.

\section{Hecken als Lebensraum}

Hecken sind ökologisch sehr wertvoll und stellen schützens- und erhaltenswerte Biotope dar. Leider werden trotzdem im Zuge von Flurbereinigungen

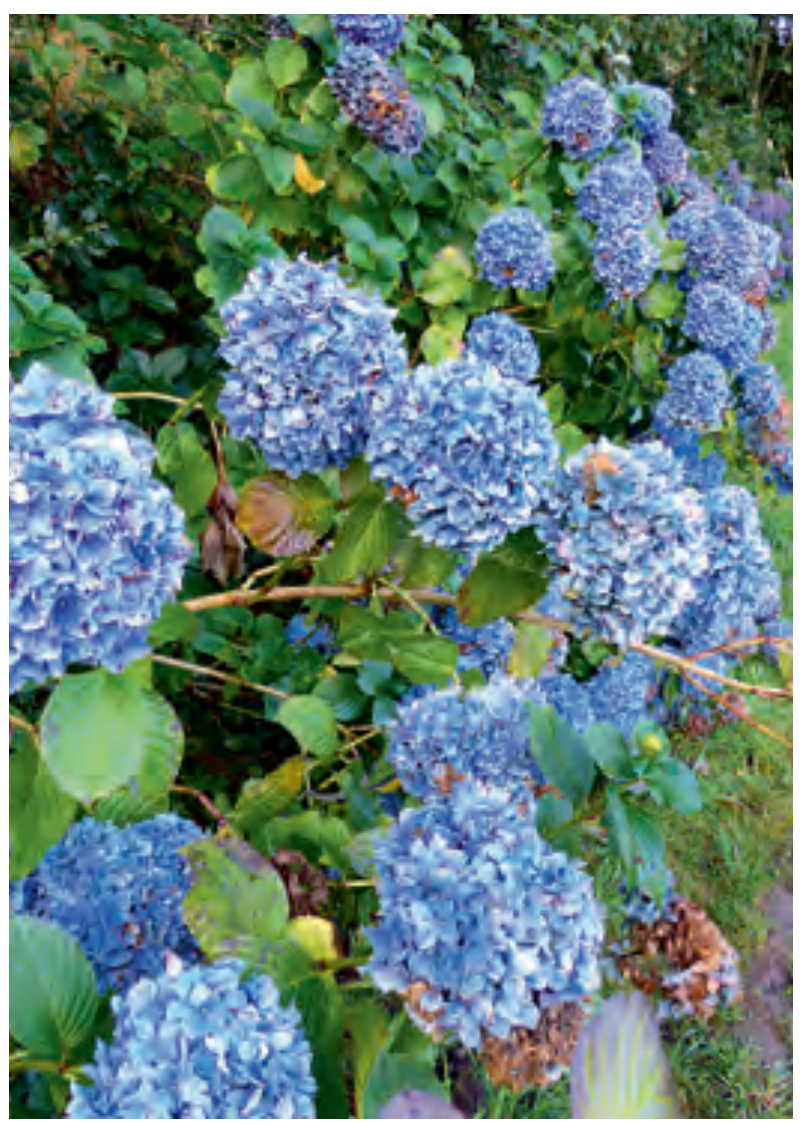

Abb. 6: In atlantisch geprägten Gebieten wie auf den Azoren oder hier in der Bretagne sind Hortensien sehr beliebte Heckenpflanzen. (Foto: H. STEINECKe) 


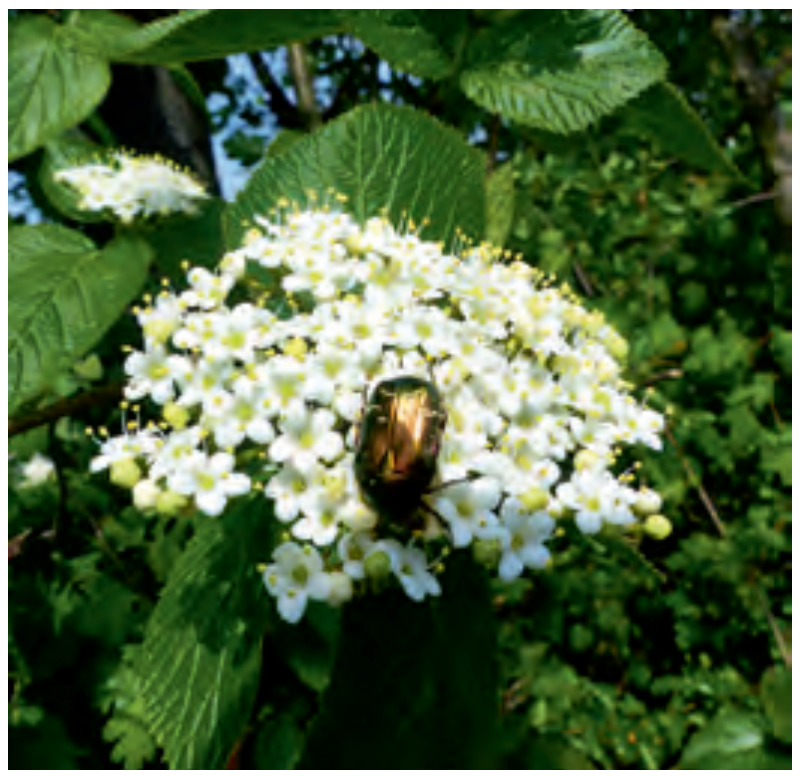

Abb. 7: Der Wollige Schneeball ist eine ideale Heckenpflanze. Seine Blüten sind für viele Insekten wie auch für Rosenkäfer eine gute Nahrungsquelle. (Foto: H. Steinecke)

immer wieder alte Hecken vernichtet. Hecken sind aber wichtig für den Erhalt einzelner Arten und auch ganzer Lebensgemeinschaften. Tiere finden hier Überwinterungsmöglichkeiten, Nahrungs-,

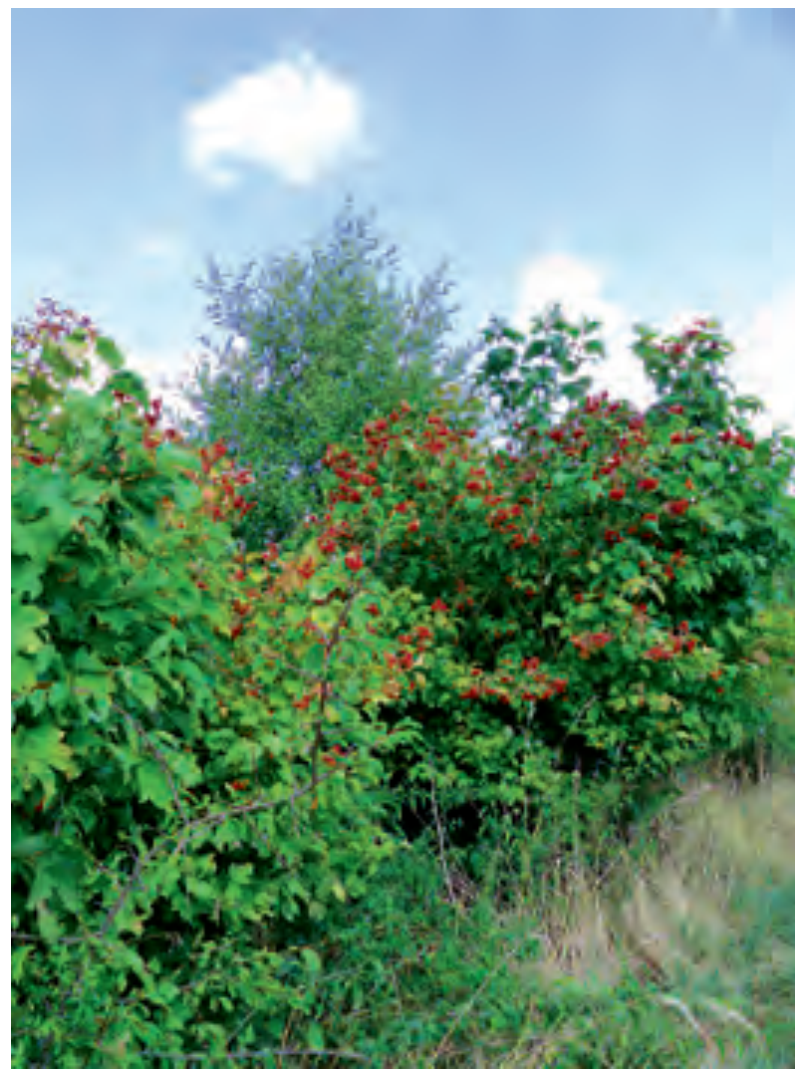

Abb. 8: Die Früchte des Gewöhnlichen Schneeballs sind bis zum Winter für viele Tiere eine willkommene Nahrung. (Foto: H. Steinecke)
Wohn-, Schlaf- und Brutraum. Für Greifvögel bieten sich Sitzgelegenheiten auf Überhältern an. Das sind Bäume, die aus der Hecke nach oben herauswachsen. Neuntöter nutzen häufig solche Aussichtspunkte, um nach Nahrung Ausschau zu halten. Auf den Dornen spießen sie ihre Beute auf (LfL 2015).

An der Südseite der Hecke, wo es relativ wärmer und meist auch windstiller ist, fühlen sich Tiere bereits zeitig im Frühjahr und noch im kühler werdenden Herbst besonders wohl. In Hecken leben auch viele Nützlinge, die sich von dort aus in die Landschaft begeben wie z. B. Marienkäfer und Schwebfliegen (Westphal 2011).

Hecken sind wichtige Rückzugsorte für viele Tiere; bis zu 1500 Tierarten finden bei uns in Hecken einen geeigneten Lebensraum. Die in Hecken lebenden Tiere entfernen sich unterschiedlich weit von diesen. Bestimmte Laufkäfer sind im Umkreis der Hecke von nur rund $50 \mathrm{~m}$ anzutreffen, während sich Igel etwa 250 m entfernen. (LfL 2015)

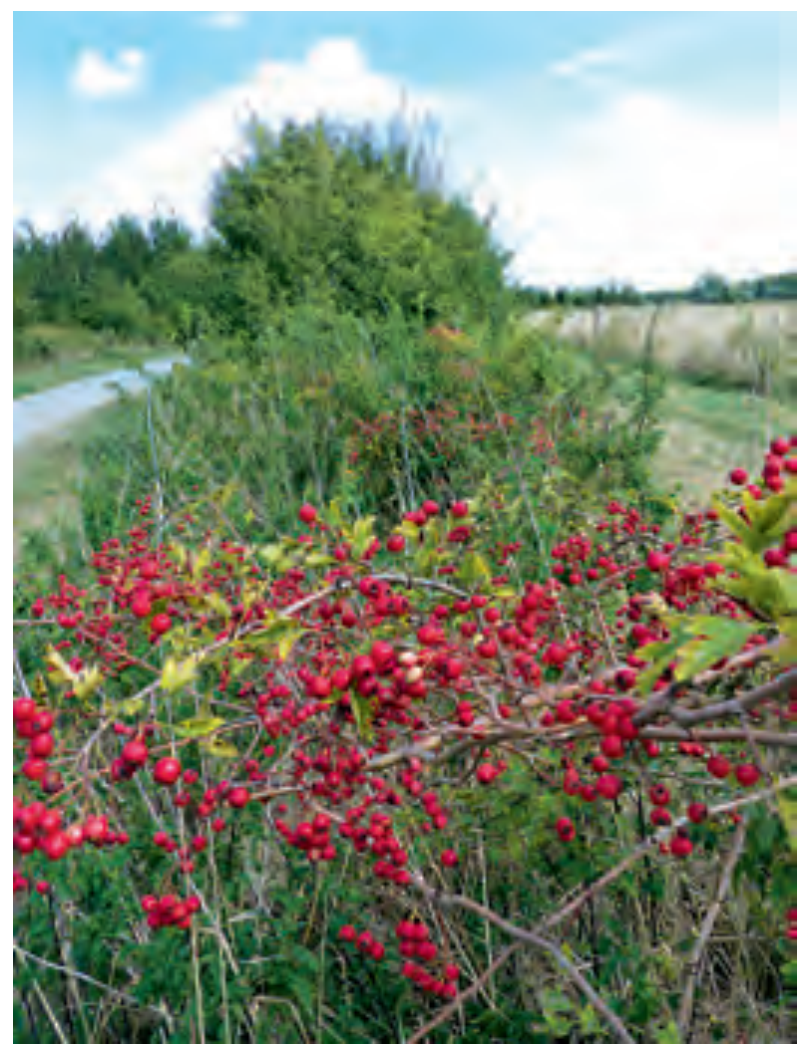

Abb. 9: Weiß- oder Hagedorn ist als Heckenpflanze und Vogelschutzgehölz sehr gut geeignet. Die sehr sauren und herben Früchte wurden früher in Notzeiten gegessen, sie sind auch zu Marmelade verarbeitbar. (Foto: H. STEInEcke) 


\section{Benjeshecke}

Diesen Heckentyp hat der deutsche, bei Bremen lebende Landschaftsgärtner, Naturfotograf und Schriftsteller Hermann Benjes (1937-2007) in den 1980er Jahren beschrieben, weiterentwickelt und in unzähligen Vorträgen publik gemacht. Sie werden meist in der freien Landschaft angelegt durch lockeres Aufschichten von Gehölzschnitt. Daraus entwickelt sich von ganz alleine eine Hecke. Über den Wind werden Nährstoffe und Samen herangeweht, und auch über den Kot von Vögeln und die darin enthaltenen Samen siedeln sich immer neue Pflanzenarten an.

Für die Anlage einer Benjeshecke bietet sich die Zeit zwischen Herbst und Frühjahr an. Im Idealfall wird der Gehölzschnitt auf einer Länge von maximal vier Metern sowie einer Breite und Höhe von jeweils einem Meter ausgebracht. Durch Samenanflug kann sich hier auf natürliche Weise eine Hecke entwickeln. In ihr finden wie bei allen Hecken viele verschiedene Tierarten Unterschlupf, die zum weiteren Gedeihen der Hecke beitragen, indem sie Pflanzen, Früchte und Samen verschiedenster Arten einschleppen. Es sollte beim Anlegen der Hecke darauf geachtet werden, kein Schnittgut zu verwenden, das lange nach dem Abschnitt noch in der Lage ist, wieder auszuschlagen wie die Brombeeren, da diese sich dann zu dominant entwickeln würden. Eine Zersetzung von Totholz ist außerdem erwünscht. Denn erst durch die Abbauvorgänge mitsamt der daran beteiligten Kleinstlebewesen und der entsprechenden Humusbildung werden optimale Entwicklungsbedingungen für neue sich etablierende Gehölze geschaffen. Eine Benjeshecke anzulegen ist einfach und kostengünstig und deshalb empfehlenswert.

\section{Knick- und Wallhecke}

Die für Schleswig- Holstein charakteristischen Knick-Hecken oder Knicks werden auch Wallhecken genannt. Knicks nehmen etwa ein Prozent der Landschaftsfläche in diesem nördlichen Bundesland ein. Knick-Hecken gedeihen häufig auf bis einen Meter hohen Erdwällen. Schon vor 200 Jahren wurden diese Hecken zur Abgrenzung der landwirtschaftlichen Flurstücke angelegt. Als

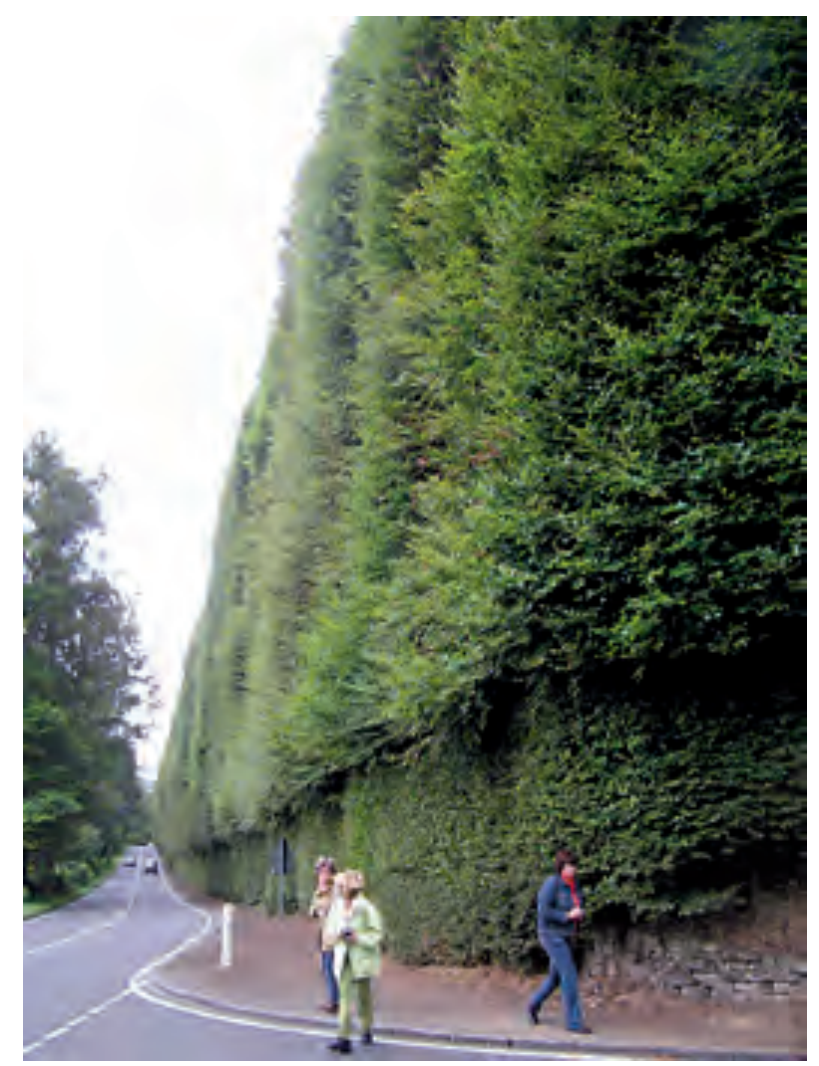

Abb. 10: Hecken können gewaltige Ausmaße erreichen. Die Meikleour Beech Hedge wurde 1745 gepflanzt und besteht aus Rot-Buchen. Sie ist bis $36 \mathrm{~m}$ hoch. Seit 1966 ist sie im Guinessbuch der Rekorde als höchste Hecke der Welt verzeichnet. Mit einer Länge von $530 \mathrm{~m}$ ist sie zudem die längste Hecke in Großbritannien. Sie wird alle 10 Jahre geschnitten und dabei neu vermessen. (Foto: H. Steinecke).

Korridor verbinden Knicks verschiedene zerstreut voneinander befindliche Lebensräume. Schlehen, Haselsträucher und Hainbuche sind klassische Gehölzarten dieser Hecken. Damit sie schnell dicht werden, werden sie im Winter seitlich geschnitten und müssen zu lang herausstehende Zweige umgeknickt werden. Daraus leitet sich der Name Knick ab. Knicks stehen unter Naturschutz.

\section{Heckengehölze}

Bei der Wahl von Heckengehölzen sollte man sich an der Umgebung, in der sie gepflanzt werden, orientieren. Die Artenauswahl hängt auch vom Nutzungszweck (z. B. lebender Zaun, Brennholzlieferant) ab. Durch den Windschutz, den eine Hecke in der freien Landschaft bietet, führt dies in der Landwirtschaft zu einer besseren Entwicklung von Nutzpflanzen. Wachsen Hecken in Hauptwindrichtung, wird die Windgeschwindigkeit um gut die Hälfte vermindert. Für manche Landschaften (Europas) sind bestimmte Hecken äußerst charak- 


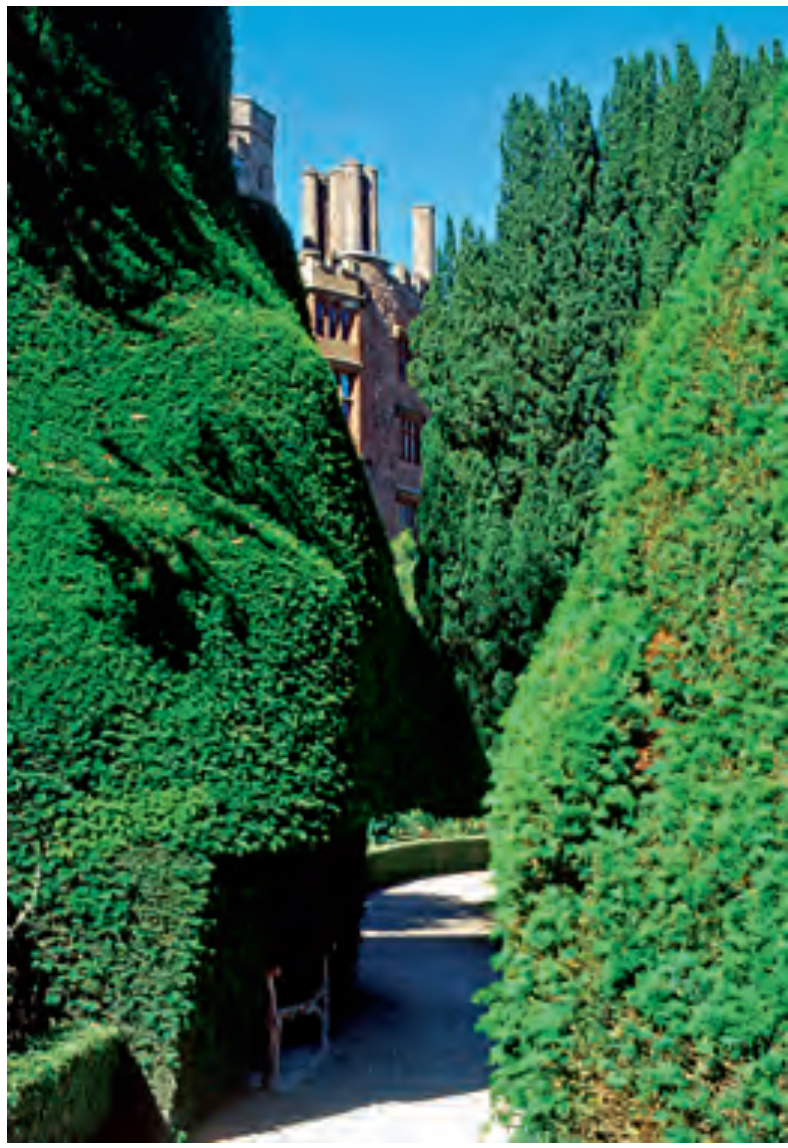

Abb. 11: Weil sie sich so gut schneiden lassen und immer wieder buschig austreiben, sind Eiben seit langer Zeit beliebte Heckenpflanzen, auch für Parks und Gärten. Hier eine alte Eibenhecke im Park von Powis Castle in Wales. (Foto: P. SCHUBERT)

teristisch. In Großbritannien oder Irland findet man häufig ausgesprochen lange und üppige Hecken. Sie enthalten häufig die dort als Neophyten weit verbreiteten Fuchsien (Fuchsia magellanica). Auch Eiben, die sich leicht schneiden lassen und dabei sehr dicht werden, sind sehr beliebt. Auf den meist sehr windigen Azoren werden fast alle landwirtschaftlichen Flächen von langen, breiten Hecken eingefriedet, um die hier oft sehr stark wehenden Winde abzuschwächen. Im Sommer dominieren hier mitunter lange, meist blau blühende Hortensienhecken die Landschaft.

Bei uns werden für die Anlage von Hecken naturnahe Pflanzungen mit einheimischen Gehölzen empfohlen. Geeignete Baumarten, die sich auch leicht zurückschneiden lassen, sind Hainbuche und Feldahorn. Wer gerne Früchte ernten oder Tieren Futterpflanzen anbieten möchte, dem seien zusätzlich Holunder, Kornelkirsche, Schnee- ball, Heckenrosen, Weißdorn, Schlehe, Roter Hartriegel und Hasel empfohlen.

Hecken bereichern also unsere Landschaft deutlich, sind für den Erhalt der Artenvielfalt von großer Bedeutung und sollten erhalten bzw. auch immer wieder neu angelegt werden, auch im privaten Bereich.

\section{Literatur}

Bayerische Landesanstalt für Landwirtschaft, LfL (Hrsg) 2015: Hecken, Feldgehölze und Feldraine in unserer Landschaft. Freising-Weihenstephan. https://www.lfl.bayern.de/mam/ $\mathrm{cms}$ 07/publikationen/daten/informationen/hecken_feldgehoelze_feldraine_lfl-information.pdf

Kurz, P., Machatschek, M. \& Igelhauser, B. 2011: Hecken. - Graz.

Perger, K. Ritter von 1864: Deutsche Pflanzensagen. Stuttgart, Oehringen. - Reprint Wiesbaden.

Schubert, P. 2004: Was sind Zauber- und Hexenpflanzen? In: Druidenfuß und Hexensessel. Magische Pflanzen. Palmengarten Sonderheft 38: 7-13. - Frankfurt am Main.

Westphal, U. 2011: Hecken - Lebensräume in Garten und Landschaft: Ökologie, Artenvielfalt, Praxis. - Darmstadt.

\section{Internetseiten}

https://de.wikipedia.org/wiki/Hag

https://de.wikipedia.org/wiki/Rheingauer_Geb\%C3\%BCck http://www.tierchenwelt.de/specials/tierleben/2782-tiere-der-hecke.html

https://de.wikipedia.org/wiki/Hermann_Benjes

\section{Anschrift der Autorin:}

Stephanie Seitz, Palmengarten Frankfurt, Siesmayerstraße 61,60323 Frankfurt, E-Mail: stephanie.seitz@stadt-frankfurt.de 